\title{
Pioglitazone up-regulates MALAT1 and promotes the proliferation of endothelial progenitor cells by increas- ing c-Myc expression in type 2 diabetes mellitus
}

\author{
Chen $\mathrm{Hu}^{\mathrm{a}}$, Yan Sun ${ }^{\mathrm{b}}$, Xiaoxu Yang, \\ ${ }^{a}$ Department of Pharmacy, Tianjin First Center Hospital, Tianjin, 300912, China. \\ ${ }^{b}$ Department of Pharmacy, Nankai Hospital, Tianjin, 300100, China. \\ ' Department of Pharmacy, Tianjin Medical University Chu Hisen-I Memorial Hospital, Tianjin, 300400, China.
}

\begin{abstract}
Background: Evidence suggests that pioglitazone improves the function of endothelial progenitor cells (EPCs); however, knowledge of its molecular mechanism remains limited. In the present study, the role of long noncoding RNA MATAL1 in modulating the number and function of EPCS in type 2 diabetes mellitus (T2DM) was characterized.

Methods: Circulating EPCs were obtained from the peripheral blood of healthy subjects and T2DM patients with or without pioglitazone treatment. The in vitro experiments were conducted in bone marrow-EPCs to evaluate the effect of pioglitazone and its possible mechanism.

Results: Pioglitazone increased the number of circulating EPCs and improved their function in T2DM patients. By transfecting the EPCs with siRNA-MALAT1, the increase in c-Myc protein expression induced by pioglitazone treatment was canceled. In $\mathrm{db} / \mathrm{db}$ diabetic mice, a glucose tolerance test showed that pioglitazone increased glucose tolerance, which was reversed by siRNA-MALAT1.

Conclusion: Pioglitazone improves EPC function, possibly through regulating MALAT1 and c-Myc expression in T2DM.
\end{abstract}

Keywords: Pioglitazone, diabetes mellitus, MALAT1, endothelial function, glucose tolerant

\section{Introduction}

Pioglitazone, a peroxisome proliferator-activated receptorgamma (PPAR $\gamma$ ) agonist, is an insulin-sensitizing agent acting as a prescription drug for the treatment of type 2 diabetes mellitus (T2DM) $[1,2]$. It has been demonstrated that pioglitazone provides benefits in a diverse population of patients with diabetes, lowering the risk of death, myocardial infarction, or stroke [3]. A subset of stem cells called endothelial progenitor cells (EPCs) exist with the

\footnotetext{
* Corresponding author : Xiaoxu Yang

Mailing address: Department of Pharmacy, Tianjin Medical University Chu Hisen-I Memorial Hospital, Address: No.6 North Huanrui Rd, Beichen District, Tianjin, People's Republic of China.

Email: yangxiaoxu729@163.com
}

Received: 18 February 2020 / Accepted: 13 March 2020 vascular circulation and play important roles in the maintenance of endothelial homeostasis and vascular integrity, contributing to vessel repair following endothelial damage [4]. Reduced EPC number and function are associated with the presence of cardiovascular events, including T2DM [5-7]. Pioglitazone involves controlling blood glucose through insulin-sensitizing effects [8]. In addition, emerging evidence indicates that improvements in endothelium-dependent vascular function also contributes to the protective effect of pioglitazone on T2DM and its associated complications [9]. In the last decade, the role of pioglitazone in EPCs has been highlighted. Spigoni et al. showed that pioglitazone treatment improves the in vitro viability and function of EPCs and reduces cardiovascular risk in impaired glucose tolerant individuals independent of the insulin-sensitizing action [10], and the PI3K/Akt signal pathway has been proposed in this process [11]. However, knowledge remains limited on the molecular mechanisms of pioglitazone in EPC regulation.

Non-coding RNAs have been widely considered to take part in various pathophysiologic processes of animal and 
human diseases $[12,13]$. Long non-coding RNA (IncRNA) has more than 200 nucleotides, is a type of novel non-coding RNA, and participates in the modulation of gene transcription, post-transcriptional regulation, and epigenetic regulation [14, 15]. Metastasis-associated lung adenocarcinoma transcript 1 (MALAT 1), also known as noncoding nuclear-enriched abundant transcript 2 (NEAT2), was first found in lung cancer and regulates the expression of metastasis-associated genes [16, 17]. A newly published paper showed that MALAT1 controls a phenotypic switch in endothelial cell and regulates its function and vessel growth [18]. It has also been demonstrated that MALAT1 plays a pathogenic role in endothelial cell dysfunction in diabetes mellitus [19]. However, whether MALAT1 is involved in regulating EPC function in T2DM remains unknown. The objective of this study was to examine the effect of pioglitazone on EPC function and evaluate whether MALAT1 participates in this process.

\section{Materials and methods}

\section{Subjects}

The human study was designed as a prospective clinical trial of healthy subjects and patients with T2DM. All participants were recruited from Tianjin Medical University Chu Hisen-I Memorial Hospital. The trial was performed with the approval of the Ethics Committee of Tianjin Medical University Chu Hisen-I Memorial Hospital. Informed consent was obtained from all participants.

Thirty-two healthy subjects (control) and 65 T2DM patients were recruited. Thirty-two patients with T2DM received a treatment of pioglitazone (30 mg/day) for four weeks, and the other 33 patients received no treatment. In this study, T2DM was defined according to the American Diabetes Association criteria [20]. Patient with one or more of the following criteria were excluded: type 1 diabetes, treatment with insulin, treatment with diet and/or exercise, treatment with any thiazolidinedione, and treatment with medications known to affect EPC biology. Four weeks after treatment, fasting venous blood was collected. The fresh whole blood was used to assess the number and function of the circulating EPCs. The serum was obtained to perform the routine examination.

\section{Flow cytometry analysis of EPC number}

Hematopoietic stem cells (CD34 and CD133) and endothelial cells (KDR) are the surface markers of EPCs, and the determination of these three markers represents the number of EPCs. Flow cytometry was used to determine the expression of CD34, CD133, and KDR in mononuclear cells (MNCs). A volume of $10 \mathrm{~mL}$ fresh peripheral venous blood treated with heparin $(20 \mathrm{u} / \mathrm{mL})$ was used to obtain MNCs via a Ficoll-Hypaque Solution (Tianjin Hanyang Biologicals Technology Co., Ltd, China). The MNCs were then re-suspended in a M199 medium (Hyclone, USA) and diluted in a FACS buffer for the measurement of CD34, CD133, and KDR via incubation with
anti-CD34-PC-5 (Becton Dickinson, USA), anti-CD133FITC (Beijing Bo Orson Biological Technology Co., Ltd., China), and anti-KDR-PE (R\&D Systems, USA) in the dark. Cells were washed twice with the FACS buffer and fixed with $2 \%$ paraformaldehyde. Analysis was performed via the assessment of 100,000 events for each separate examination. The expression of CD34, CD133, and KDR was reported as a percentage of the total events.

\section{EPC adhesion}

Dissociated adherent cells with $0.25 \%$ trypsin and resuspended them in the medium. The cell suspension was washed with PBS for 5 min. $1 \times 10^{5}$ cells were seeded in a fibronectin-coated culture plate for $30 \mathrm{~min}$. The adhesion cells were counted under a light microscope.

\section{Migration assay}

Boyden chambers were used to assess the migration of the EPCs, which were treated in a Boyden chamber (Haimen Qi LinBeiEr instrument manufacturing co., LTD., China) with ultraviolet irradiation overnight. Cells were dissociated with trypsin $(200 \mu \mathrm{L} /$ well $)$ and re-suspended in a M199 medium with $5 \%$ fetal bovine serum. The concentration of cells was adjusted to $2.0 \times 10^{5}$ cells/well. The lower chamber was added to a M199 medium containing $20 \mu \mathrm{L}$ vascular endothelial growth factor (VEGF, $50 \mathrm{ng} / \mathrm{mL}$ ). Placed $200 \mu \mathrm{L}$ cell suspension in the upper chamber with polycarbonate-free membrane $(8.0 \mu \mathrm{m}$ proes $)$ to culture for $90 \mathrm{~min}$. The cells were then removed from the upper chamber, and the migrated cells were stained on the lower surface of the membrane with haematoxylin and eoxsin. The cells were then counted under the microscope.

\section{In vitro angiogenesis assays}

The angiogenic capacity of the EPCs was measured by the in vitro Angiogenesis Assay Kit (Chemicon, USA) according to the manufacturer's instructions. Cells were harvested with trypsin and cultured in a 96-well plate precoated with ECMatrix gel for $24 \mathrm{~h}$. The cells were then counted under a microscope, and tube formation was calculated.

\section{Bone marrow-EPC culture}

In vitro experiments were performed using a bone marrow-EPC culture. Healthy male C57BL/6 mice aged 8-10 weeks were sacrificed after anesthetization with ketamine $(25 \mathrm{mg} / \mathrm{kg})$. Femurs and tibias were dissected, and the adhering tissues were completely removed. Both ends of the bones were excised. Bone marrow cells (BMCs) were harvested via flushing with Endothelial Cell Growth Medium 2 (EGM-2, Lonza, USA) and the density gradient centrifugation method. The cells were then re-suspended with EGM-2 containing 20\% fetal calf serum (FCS), and the cell suspension $\left(1 \times 10^{6}\right.$ cells $\left./ \mathrm{mL}\right)$ was seeded in a culture plate at $37^{\circ} \mathrm{C}$ with $5 \% \mathrm{CO}_{2}$. Adherent cells were incubated with fresh EGM-2 three days after removing the unattached cells. The medium was replaced every two days, and the morphology of the cells was monitored. When the 


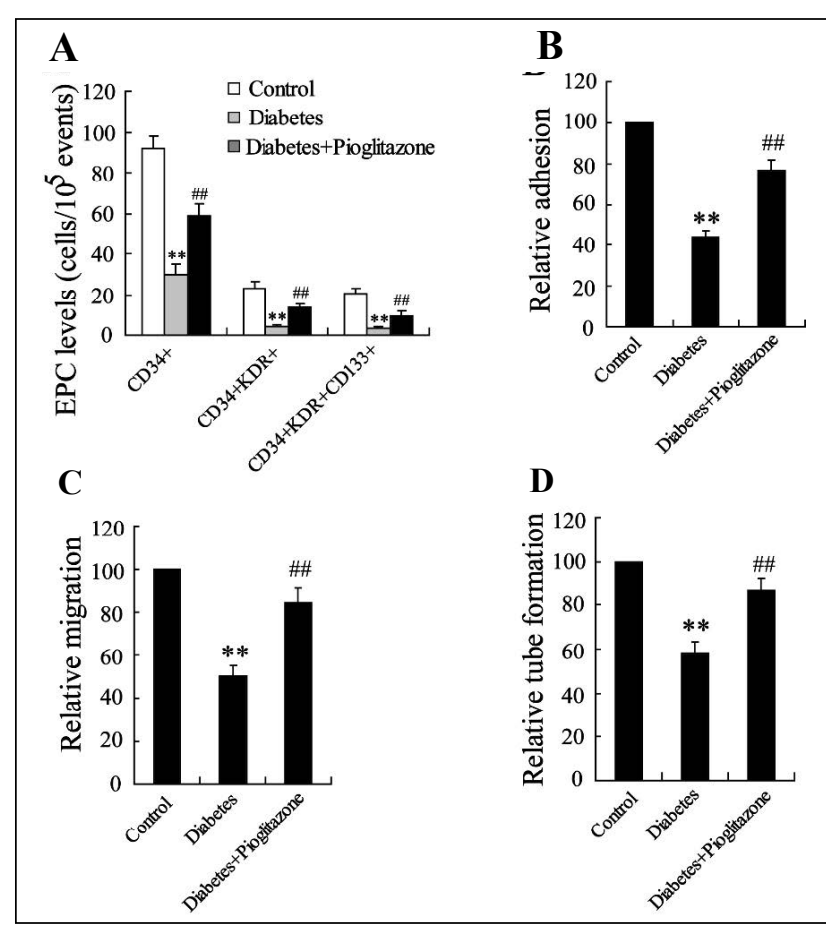

Figure 1. Effects of pioglitazone on the number and function of circulating EPCs in T2DM patients. EPC number (A), adhesion (B), migration (C) and tube formation (D) were detected in healthy subjects or T2DM patients with or without the treatment of pioglitazone $(30 \mathrm{mg}$ pd for four weeks). $* * P<.0 .01$, vs. control; \#\# $P<0.01$, vs. diabetes.

cells had grown to $80 \%$ confluence (days 7 to 10 ), $0.125 \%$ trypsin was used for cell dissociation.

\section{RNA extraction and quantitative real-time PCR}

The circulating EPCs or bone marrow-EPCs were lysed with a TRIzol reagent (Invitrogen, USA), and total RNA was extracted according to the manufacturer's instruction. The PrimeScriptTM RT-PCR Kit (Takara, China) was used to synthesize the cDNA with RNA. For the quantitative analysis of MALAT1 and c-Myc, the BIORAD CFX96 touch q-PCR system was applied with a IQ SYBR Green supermix (Bio-Rad Laboratories, USA). All samples were read in triplicate, and the relative expression values were normalized to GAPDH and $\beta$-actin expression via the $2^{-\Delta \Delta C T}$ method, respectively.

\section{Western blot}

For western blot analysis, the cells were harvested and washed twice in cold PBS. Then, they were lysed in a RIPA lysis buffer (50 mM Tris- $\mathrm{HCl}, \mathrm{pH} 7.4,150 \mathrm{mM}$ $\mathrm{NaCl}, 1 \%$ Triton $\mathrm{X}-100$, and $0.5 \%$ sodium deoxycholate, $0.1 \%$ sodium dodecyl sulfate) supplemented with a protease inhibitor for $1 \mathrm{~h}$. The lysates were then centrifuged at $10,000 \times \mathrm{g}$ for $10 \mathrm{~min}$ at $4{ }^{\circ} \mathrm{C}$, the supernatants were collected. Protein concentrations were measured by the Bradford Protein Assay Kit (Beyotime, China). Equal amounts of protein extract for each sample were loaded on $10 \%$ SDS-PAGE electrophoresis for separation, and then the proteins were transferred to PVDF membranes (Millipore, USA) and blocked in 5\% nonfat milk prior to incubation with the primary antibodies against c-Myc and $\beta$-actin (1:1000, Cell Signaling Technology, USA) overnight at $4{ }^{\circ} \mathrm{C}$. A HRP-conjugated secondary antibody (1:5000, Cell Signaling Technology, USA) was then incubated in a membrane for $90 \mathrm{~min}$.

\section{Animal experiments}

$\mathrm{C} 57 \mathrm{BL} / 6 \mathrm{~J} \mathrm{db} / \mathrm{db}$ diabetic mice at ten weeks of age were purchased from the Model Animal Research Center of Nanjing University (Nanjing, China). All animals were fed with a standard chow diet in a temperature-controlled room. Isolated autologous EPCs from mice were used to treat diabetic mice. Before injection, EPCs were pre-incubated with $10 \mathrm{mM}$ Pioglitazone for $2 \mathrm{~h}$ and transfected with pLVX-IRES-ZsGreenl-si-MALAT1 (pLVX-IRESZsGreenl as control) for $24 \mathrm{~h}$. The EPCs were intravenously injected into the mice once a week for 16 weeks. A glucose tolerance test was performed to determine glucose tolerance. After fasting for 15 hours, the mice received $0.5 \mathrm{~g} / \mathrm{kg}$ glucose via intraperitoneal injection. The blood was then collected from the tail vein, and the blood glucose concentration was measured. The animal experiments were conducted according to the Guide for the Care and Use of Laboratory Animals and approved by the animal experimental ethics committee of Tianjin Medical University Chu Hisen-I Memorial Hospital.

\section{Statistical analysis}

All statistical analyses were performed using SPSS 13.0 statistic software, and the data was represented as mean \pm standard error (SEM). The difference in the means between the two groups was analyzed by Student's t test. A $P$-value less than 0.05 was regarded as statistically significant.

\section{Results}

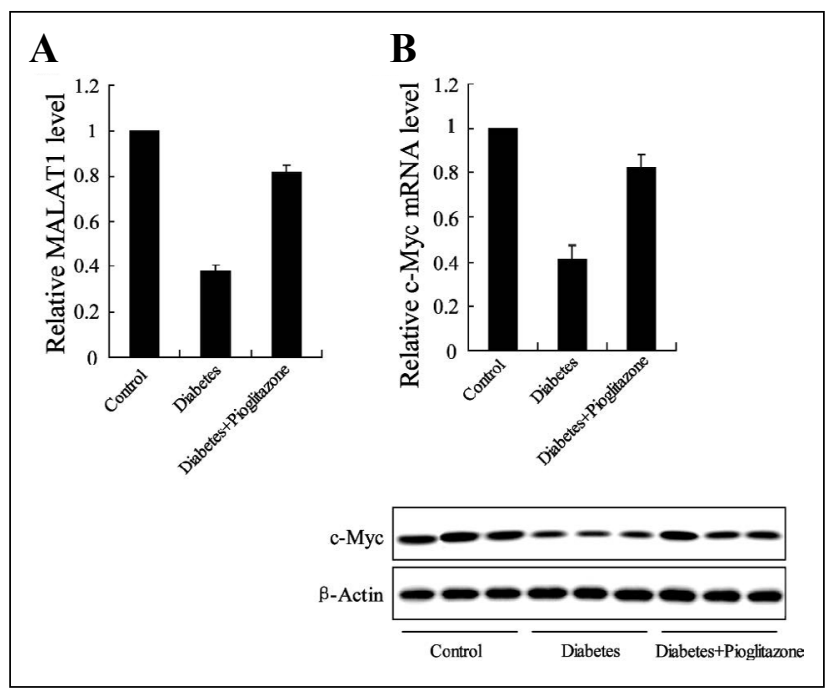

Figure 2. Effects of pioglitazone on MALAT1 and the c-Myc expression of circulating EPCs in T2DM patients. The expression levels of MALAT1 RNA (A) and c-Myc mRNA and protein (B) were determined in healthy subjects or T2DM patients with or without the treatment of pioglitazone (30 mg pd for four weeks). ${ }^{* *} P<0.01$, vs. control; \#\# $P<$ 0.01 , vs. diabetes. 


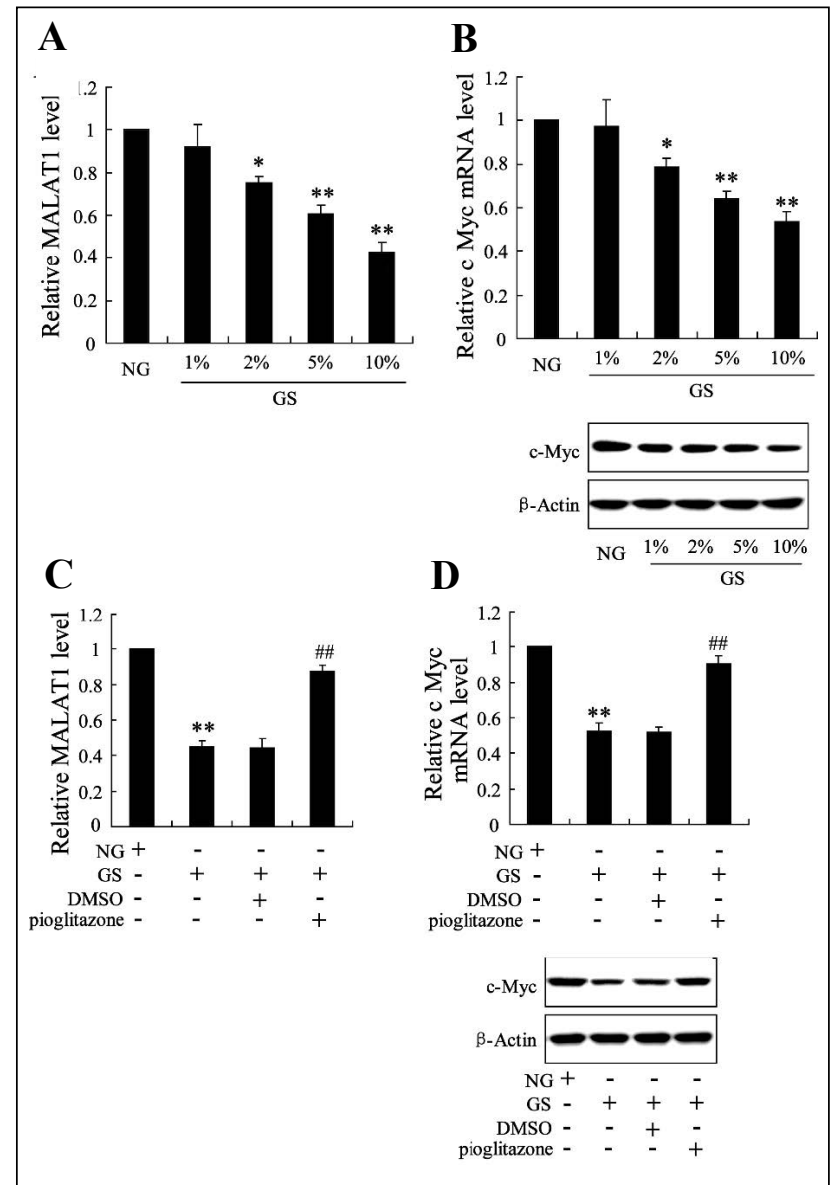

Figure 3. Effects of pioglitazone on MALAT1 and c-Myc expression in bone marrow-EPCs exposed to high glucose in vitro. The expression levels of MALAT1 RNA (A) and c-Myc mRNA and protein (B) were determined in bone marrow EPCs with different concentrations of glucose $(1 \%, 1 \%, 2 \%, 5 \%$, and 10\%). These expressions $(\mathbf{C}, \mathbf{D})$ was also determined in high glucose $(10 \%)$-induced bone marrow-EPCs with the treatment of pioglitazone. $* * P<0.01$, vs. control; \#\# $P<0.01$, vs. diabetes.

Pioglitazone improves the function of circulating EPCs in T2DM patients.

The number and function of the circulating EPCs in T2DM patients were first evaluated. As expected, T2DM patients exhibited decreased EPC markers CD34+, $\mathrm{CD} 34+\mathrm{KDR}+$, and CD34+KDR+CD133+ numbers compared with healthy subjects (Figure 1A). The function of the circulating EPCs was then measured. The results showed that the migration, adhesion, and formation of the EPCs were reduced to $43.4 \%, 50.9 \%$ and $58.1 \%$ in the T2DM patients, respectively (Figure 1B-1D). Administrated with $30 \mathrm{mg}$ pioglitazone for 4 weeks, the number and function of circulating EPCs in T2DM were significantly elevated.

\section{MALAT1 and c-Myc expression of circulating EPCs in T2DM patients}

The MALAT1 level of the circulating EPCs was then investigated in the T2DM patients, and the results showed that it had significantly down-regulated to $38.3 \%$, whereas the pioglitazone treatment resulted in an increase of the
MALAT1 expression level to $81.3 \%$ (Figure 2A). c-Myc has been demonstrated to play a vital role in adequate angiogenesis and regulating endothelial dysfunction [21, 22]. The c-Myc mRNA and protein expression levels of the circulating EPCs in the T2DM patients presented a similar trend with respect to MALAT1, while the pioglitazone treatment up-regulated these expressions (Figure 2B).

Pioglitazone affected the MALAT1 and c-Myc expression in bone marrow-EPCs exposed to high glucose

The effects of pioglitazone in the in vitro model of T2DM were demonstrated. Co-incubated bone marrow-EPCs with different concentrations of glucose $(1 \%, 2 \%, 5 \%$ and $10 \%$ ) as well as a decreasing MALAT1 and c-Myc mRNA and protein expression were shown in a dose-dependent manner (Figure 3A and 3B). However, the decrease of MALAT1 and c-Myc expression in EPCs exposed to high glucose content $(10 \%)$ could also be reversed by the treatment of pioglitazone in vitro (Figure $3 \mathrm{C}$ and $3 \mathrm{D}$ ).

\section{MALAT1 down-regulation affected the effect of piogli- tazone on c-Myc expression}

Pioglitazone has previously been confirmed to play a role in reversing the effect of high glucose on MALAT1 and c-Myc expression in bone marrow-EPCs. Then, bone marrow-EPCs were administered si-MALAT1 for downregulating its expression, and the role of pioglitazone in increasing c-Myc protein expression was largely canceled (Figure 4A). However, the mRNA expression of c-Myc was not changed by si-MALAT1 (Figure 4B).

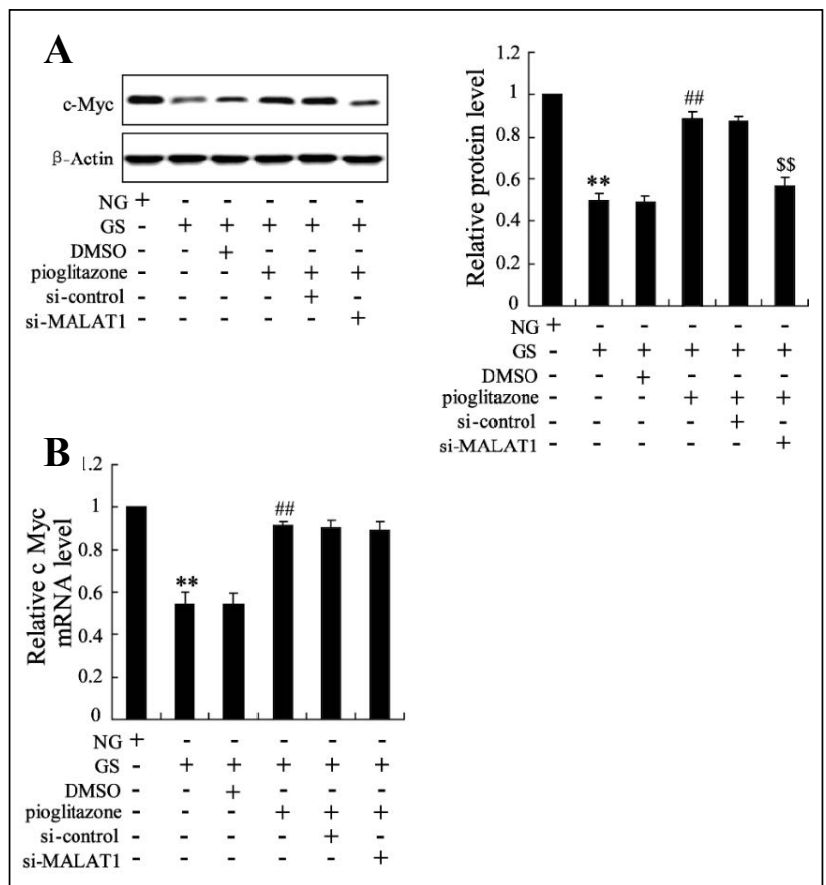

Figure 4. MALAT1 is involved in the effect of pioglitazone on c-Myc expression in bone marrow-EPCs exposed to high glucose content in vitro. The expression of $\mathrm{c}-\mathrm{Myc}$ in the protein levels (A) and mRNA levels (B) were determined in bone marrow EPCs with different treatments. ** $P<0.01$, vs. NG; \#\# $P<0.01$, vs. NG+DMSO; $\$ \$ P<0.01$, vs. NG+DMSO+si-control. 


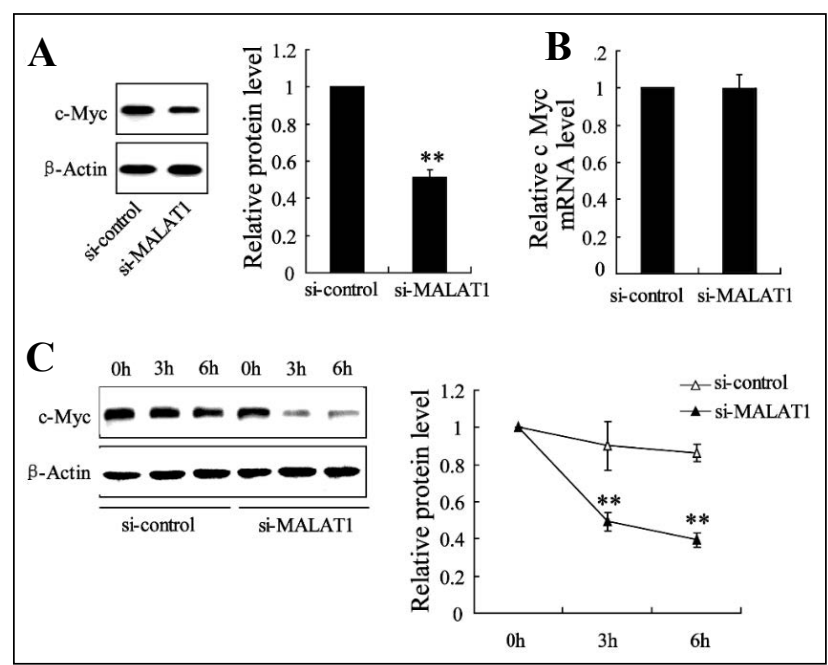

Figure 5. MALAT1 down-regulation decreased c-Myc expression in protein levels. The expression of $\mathrm{c}-\mathrm{Myc}$ in the protein levels $(\mathbf{A}, \mathbf{C})$ and in mRNA levels (B) were determined in bone marrow EPCs with the treatment of si-MALAT1 (or si-control). ${ }^{* *} P<0.01$, vs. si-control.

\section{MALAT1 down-regulation affected c-Myc expression and stability}

In order to confirm the role of MALAT1 in regulating cMyc expression, co-incubated bone marrow EPCs with si-MALAT1 alone to observe the c-Myc expression. As shown in Figure 5, c-Myc protein expression was decreased to $51.0 \%$ (Figure 5A), but its mRNA expression was not altered (Figure 5B) by si-MALAT1. In addition, c-Myc protein expression decreased at the time of siMALAT1 treatment (Figure 5C).

\section{The glucose tolerance of $\mathbf{d b} / \mathbf{d b}$ mice with the treatment of EPCs}

The therapeutic effect EPCs with the treatment of pioglitazone and si-MALAT 1 on the glucose tolerance of $\mathrm{db} / \mathrm{db}$ mice was investigated. The results showed that EPCs with the treatment of pioglitazone resulted in the improvement of glucose tolerance; however, administrated EPCs with pioglitazone and si-MALAT1, the benefit of pioglitazone for blood glucose control was largely reversed (Figure 6).

\section{Discussion}

In the present study, we found that pioglitazone significantly increased the number and function of circulating EPCs. Pre-incubated EPCs with pioglitazone and then treated $\mathrm{db} / \mathrm{db}$ diabetic mice, a significant decrease of the blood glucose levels and improvements in glucose tolerance were observed. Moreover, the up-regulation of MALTA1 expression induced by pioglitazone was proposed to take part in the increasing number and function of EPCs and as providing a benefit for the treatment of T2DM.

The EPCs co-expressed the surface markers of both hematopoietic stem cells (CD34 and CD133) and endothelial cells (KDR) [23]. The measurement of these three cell markers is commonly used to determine EPC counts. In agreement with previous observations, we demonstrated that T2DM patients had a reduced number and function of circulating EPCs [5, 24]. In the last two decades, emerging studies have illustrated that circulating EPCs act as a biomarker of endothelial function, and their alteration is characterized by a high risk of cardiovascular disease [25-27]. The impairment and reduction of EPCs are also hallmark features of type 1 and type 2 diabetes [28-30]. Autologous EPCs are considered to be a novel cellular therapy for diabetic vascular complications [31]. However, this application is limited in diabetic individuals due to their dysfunctional circulating EPCs. Previous studies have confirmed that pioglitazone increases the number and function of EPCs in individuals with diabetes mellitus [32], coronary artery disease, normal glucose tolerance [33], and impaired glucose tolerance [10], contributing to the better use of EPCs in cardiovascular disease treatment. However, few studies have been shown to investigate the molecular mechanism of pioglitazone in EPC regulation.

LncRNAs are implicated in diverse human diseases, especially in cancer, cardiovascular disorders, and immunological diseases [34]. MALAT1 was first reported in lung cancer and is associated with metastasis, migration, tumor growth, and cell proliferation in various cancers $[16,35$, 36]. Recently, Michalik et al. reported that MALTA1 indirectly triggers cell cycle blockade via the up-regulation of cell-cycle-related factors; enhances the migration capacity via thus far unknown mechanisms; and that the deficiency of MALAT1 leads to lower endothelial cell numbers and angiogenic defects [37]. It has also been reported that circulating MALAT1 expression was higher in acute myocardial infarction patients than that in healthy volunteers [38]. Poller et al. showed that MALAT1 participates in the process of cardiac innate immunity [39]. In the current study, a decreased MALAT1 expression was observed in the circulating EPCs of T2DM patients. Its expression was also reduced in bone marrow EPCs exposed to high glucose content in a dose-dependent manner. Considering the reduced number and function of circulating EPCs, we proposed that MALAT1 was correlated with EPC function

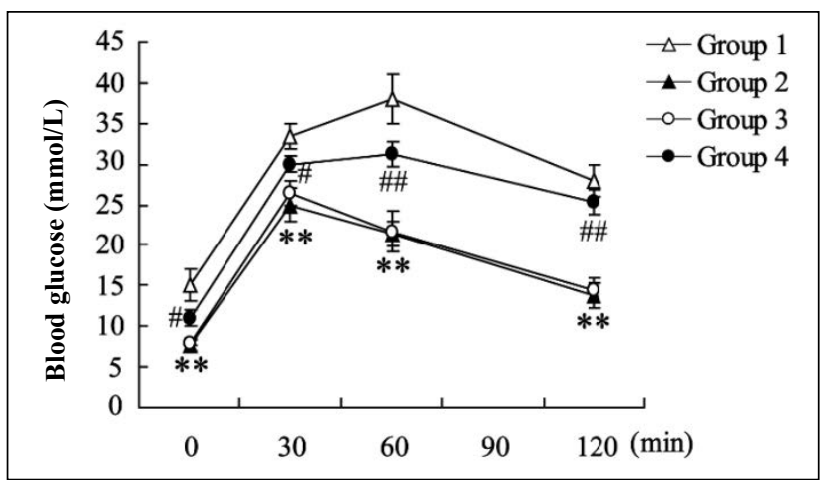

Figure 6. Effects of EPC treatment on glucose tolerance in $\mathrm{db} / \mathrm{db}$ mice. $\mathrm{The} \mathrm{db} / \mathrm{db}$ mice were administrated using autologous EPCs with different treatments. Group 1: EPCs without any treatment; Group 2: EPCs with the treatment of pioglitazone; Group 3: EPCs with the treatment of pioglitazone and pLVX-IRES-ZsGreenl (control); Group 4: EPCs with the treatment of pioglitazone and pLVX-IRES-ZsGreenlsi-MALAT1. A glucose tolerance test was then performed in the $\mathrm{db} / \mathrm{bd}$ mice. ** $P<0.01$, vs. Group 1; $\# P<0.05$, \#\# $P<0.01$, vs. Group 3. 
in T2DM patients. As expected, pioglitazone treatment contributed to the elevation of MALAT1 levels in EPCs both in vivo and in vitro.

c-Myc is a regulator gene that codes for a transcription factor and plays a critical role in cell growth, apoptosis, and metabolism in mammals [40]. It has been commonly considered to be a proto-oncogene and is vital for adequate vascular development and angiogenesis [22]. The dysregulation of c-Myc expression is closely related to cancers and cardiovascular disorders [41]. Florea et al. demonstrated that the loss of c-Myc expression in endothelial cells results in a pro-inflammatory senescent phenotype, suggesting a central regulator of endothelial dysfunction [21]. In the present study, T2DM patients exhibited the decreased c-Myc expression of circulating EPCs in mRNA and protein levels. Pioglitazone treatment up-regulated the expression of EPCs exposed to high glucose conditions both in vivo and in vitro. Previous studies have shown that c-Myc expression is essential for the transcriptional repression of p21 and p15 [42, 43]. Therefore, decreased c-Myc levels in circulating endothelial cells may lift the repression of these cell cycle inhibitors, triggering cell cycle arrest. Transfected bone marrowEPCs with si-MALAT1 for down-regulating its expression, the c-Myc expression was reduced only in protein level. In addition, si-MALAT1 largely canceled the role of pioglitazone in increasing the c-Myc protein expression of bone marrow-EPCs exposed to high glucose content. This data indicated that MALAT1 is involved in the effects of pioglitazone on EPC function via the regulation of c-Myc expression.

The main finding of this study was that MALAT1 is down-regulated in the circulating EPCs of T2DM patients and that decreased MALAT1 disrupts glucose tolerance. We also observed that pioglitazone benefits from increasing the number and function of EPCs and controlling blood glucose as well as that MALAT1 down-regulation greatly affects the effects of pioglitazone by modulating c-Myc expression. These findings suggest that autologous EPCs with pioglitazone administration hold great promise in the treatment of diabetes mellitus and diabetic vascular complications. Thus, MALAT1 may act as a new molecular target for modulating EPC function.

\section{Declaration}

Conflict of interest: The authors declare that they have no conflict of interest.

\section{References}

1. Ohira M, Yamaguchi T, Saiki A, et al. Pioglitazone improves the cardio-ankle vascular index in patients with type 2 diabetes mellitus treated with metformin. Diabetes, metabolic syndrome and obesity: targets and therapy, 2014, 7: 313.
2. Erdmann E, Spanheimer R, Charbonnel B, et al. Pioglitazone and the risk of cardiovascular events in patients with Type 2 diabetes receiving concomitant treatment with nitrates, renin-angiotensin system blockers, or insulin: results from the PROactive study (PROactive 20). Journal of diabetes, 2010, 2(3): 212-220.

3. Lincoff A M, Wolski K, Nicholls S J, et al. Pioglitazone and risk of cardiovascular events in patients with type 2 diabetes mellitus: a meta-analysis of randomized trials. Jama, 2007, 298(10): 1180-1188.

4. Kirton J P, Xu Q. Endothelial precursors in vascular repair. Microvascular research, 2010, 79(3): 193-199.

5. António N, Fernandes R, Soares A, et al. Reduced levels of circulating endothelial progenitor cells in acute myocardial infarction patients with diabetes or pre-diabetes: accompanying the glycemic continuum. Cardiovascular diabetology, 2014, 13(1): 101.

6. Vasa M, Fichtlscherer S, Aicher A, et al. Number and migratory activity of circulating endothelial progenitor cells inversely correlate with risk factors for coronary artery disease. Circulation research, 2001, 89(1): e1-e7.

7. Liao Y, Chen L L, Zeng T, et al. Number of circulating endothelial progenitor cells as a marker of vascular endothelial function for type 2 diabetes. Vascular medicine, 2010, 15(4): 279-285.

8. Miyazaki Y, Matsuda M, DeFronzo R A. Dose-response effect of pioglitazone on insulin sensitivity and insulin secretion in type 2 diabetes. Diabetes care, 2002, 25(3): 517-523.

9. Pfützner A, Marx N, Lübben G, et al. Improvement of cardiovascular risk markers by pioglitazone is independent from glycemic control: results from the pioneer study. Journal of the American College of Cardiology, 2005, 45(12): 1925-1931.

10. Spigoni V, Picconi A, Cito M, et al. Pioglitazone improves in vitro viability and function of endothelial progenitor cells from individuals with impaired glucose tolerance. PLoS One, 2012, 7(11).

11. Gensch C, Clever Y P, Werner C, et al. The PPAR- $\gamma$ agonist pioglitazone increases neoangiogenesis and prevents apoptosis of endothelial progenitor cells. Atherosclerosis, 2007, 192(1): 67-74.

12. Eddy S R. Non-coding RNA genes and the modern RNA world. Nature Reviews Genetics, 2001, 2(12): 919-929.

13. Esteller M. Non-coding RNAs in human disease. Nature reviews genetics, 2011, 12(12): 861.

14. Vance K W, Sansom S N, Lee S, et al. The long non-coding RNA Paupar regulates the expression of both local and distal genes. The EMBO journal, 2014, 33(4): 296-311.

15. Mercer T R, Dinger M E, Mattick J S. Long non-coding RNAs: insights into functions. Nature reviews genetics, 2009, 10(3): 155-159.

16. Gutschner T, Hämmerle M, Eißmann M, et al. The noncoding RNA MALAT1 is a critical regulator of the metastasis phenotype of lung cancer cells. Cancer research, 2013, 73(3): 1180-1189.

17. Ji P, Diederichs S, Wang W, et al. MALAT-1, a novel noncoding RNA, and thymosin $\beta 4$ predict metastasis and 
survival in early-stage non-small cell lung cancer. Oncogene, 2003, 22(39): 8031-8041.

18. Michalik K M, You X, Manavski Y, et al. Long noncoding RNA MALAT1 regulates endothelial cell function and vessel growth. Circulation research, 2014, 114(9): 13891397.

19. Liu J Y, Yao J, Li X M, et al. Pathogenic role of IncRNAMALAT1 in endothelial cell dysfunction in diabetes mellitus. Cell death \& disease, 2014, 5(10): e1506-e1506.

20. American Diabetes Association. Standards of medical care in diabetes-2013. Diabetes care, 2013, 36(Supplement 1): S11-S66.

21. Florea V, Bhagavatula N, Simovic G, et al. c-Myc is essential to prevent endothelial pro-inflammatory senescent phenotype. PloS one, 2013, 8(9).

22. Baudino T A, McKay C, Pendeville-Samain H, et al. c-Myc is essential for vasculogenesis and angiogenesis during development and tumor progression. Genes \& development, 2002, 16(19): 2530-2543.

23. Hristov M, Erl W, Weber P C. Endothelial progenitor cells: isolation and characterization. Trends in cardiovascular medicine, 2003, 13(5): 201-206.

24. Fadini G P, Agostini C, Avogaro A. Endothelial progenitor cells and vascular biology in diabetes mellitus: current knowledge and future perspectives. Current diabetes reviews, 2005, 1(1): 41-58.

25. Sen S, McDonald S P, Coates P T H, et al. Endothelial progenitor cells: novel biomarker and promising cell therapy for cardiovascular disease. Clinical science, 2011, 120(7): 263-283.

26. Hill J M, Zalos G, Halcox J P J, et al. Circulating endothelial progenitor cells, vascular function, and cardiovascular risk. New England Journal of Medicine, 2003, 348(7): 593-600.

27. Angelidi A, Melidonis A, Protopsaltis I, et al. Endothelial progenitor cells as a cardiometabolic risk factor marker in prediabetes. Hormones, 2014, 13(2): 244-251.

28. Fadini G P, Miorin M, Facco M, et al. Circulating endothelial progenitor cells are reduced in peripheral vascular complications of type 2 diabetes mellitus. Journal of the American College of Cardiology, 2005, 45(9): 1449-1457.

29. Menegazzo L, Albiero M, Avogaro A, et al. Endothelial progenitor cells in diabetes mellitus. Biofactors, 2012, 38(3): 194-202.

30. Tepper O M, Galiano R D, Capla J M, et al. Human endothelial progenitor cells from type II diabetics exhibit impaired proliferation, adhesion, and incorporation into vascular structures. Circulation, 2002, 106(22): 2781-
2786.

31. Jarajapu Y P R, Grant M B. The promise of cell-based therapies for diabetic complications: challenges and solutions. Circulation research, 2010, 106(5): 854-869.

32. Wang $\mathrm{C} \mathrm{H}$, Ting $\mathrm{M} \mathrm{K}$, Verma $\mathrm{S}$, et al. Pioglitazone increases the numbers and improves the functional capacity of endothelial progenitor cells in patients with diabetes mellitus. American heart journal, 2006, 152(6): 1051. e11051. e8.

33. Werner C, Kamani C H, Gensch C, et al. The peroxisome proliferator-activated receptor- $\gamma$ agonist pioglitazone increases number and function of endothelial progenitor cells in patients with coronary artery disease and normal glucose tolerance. Diabetes, 2007, 56(10): 2609-2615.

34. Wapinski O, Chang H Y. Long noncoding RNAs and human disease. Trends in cell biology, 2011, 21(6): 354-361.

35. Tripathi V, Shen Z, Chakraborty A, et al. Long noncoding RNA MALAT1 controls cell cycle progression by regulating the expression of oncogenic transcription factor BMYB. PLoS genetics, 2013, 9(3).

36. Gutschner T, Hämmerle M, Diederichs S. MALAT1-a paradigm for long noncoding RNA function in cancer. Journal of molecular medicine, 2013, 91(7): 791-801.

37. Michalik K M, You X, Manavski Y, et al. Long noncoding RNA MALAT1 regulates endothelial cell function and vessel growth. Circulation research, 2014, 114(9): 13891397.

38. Vausort M, Wagner D R, Devaux Y. Long noncoding RNAs in patients with acute myocardial infarction. Circulation research, 2014, 115(7): 668-677.

39. Poller W, Gast M, Schroen B, et al. The Long Noncoding MALAT1-MascRNA System is a Novel Regulator of Cardiac Innate Immunity. Circulation, 2014, 130(suppl_2): A11247-A11247.

40. Dang C V. c-Myc target genes involved in cell growth, apoptosis, and metabolism. Molecular and cellular biology, 1999, 19(1): 1-11.

41. Nigris F, Balestrieri M L, Napoli C. Targeting c-Myc, Ras and IGF cascade to treat cancer and vascular disorders. Cell Cycle, 2006, 5(15): 1621-1628.

42. Claassen G F, Hann S R. A role for transcriptional repression of p21CIP1 by c-Myc in overcoming transforming growth factor $\beta$-induced cell-cycle arrest. Proceedings of the National Academy of Sciences, 2000, 97(17): 94989503.

43. Staller P, Peukert K, Kiermaier A, et al. Repression of p15 INK4b expression by Myc through association with Miz1. Nature cell biology, 2001, 3(4): 392-399.

Cite this article as: $\mathrm{Hu} \mathrm{C}$, Sun Y, Yang X. Pioglitazone up-regulates MALAT1 and promotes the proliferation of endothelial progenitor cells through increasing c-Myc expression in type 2 diabetes mellitus[J]. Aging Pathobiology and Therapeutics, 2020, 2(1): 38-44. 